\title{
Tendencias del orden internacional y sus efectos sobre el tablero sudamericano
}

\author{
International Order Trends and their effects on the South American chessboard
}

DOI: https://doi.org/10.22456/2178-8839.106944

\author{
María Elena Lorenzini \\ Universidad Nacional de Rosario, Rosario, Argentina \\ melorenzini@gmail.com \\ Gisela Pereyra Doval \\ Universidad Nacional de Rosario, Rosario, Argentina
}

gpdoval@gmail.com

\begin{abstract}
Resumen
Este artículo toma como punto de partida que el denominado orden liberal internacional se encuentra cuestionado por un conjunto de tendencias globales entre las cuales sobresalen: el ascenso de China, la emergencia y extensión de liderazgos políticos i-liberales, cierto grado de retroceso del libre comercio en favor de políticas menos multilaterales y más proteccionistas. La configuración de estas tendencias globales incide sobre los márgenes de maniobra que los países sudamericanos disponen para diseñar y ejecutar sus estrategias de inserción internacional. Por ello, nos proponemos identificar las tendencias globales y sub regionales más relevantes en tanto asumimos que las primeras constriñen, y habilitan, afectan y moldean en diversos grados e intensidades, las dinámicas de nuestra región. El diseño metodológico que se emplea es un abordaje bibliográfico, cualitativo, descriptivo y analítico. En virtud de lo expuesto, este artículo se inicia con la presentación de los fundamentos que dan cuenta de las tensiones que experimenta el orden liberal internacional a partir de las premisas propuestas por Haass (2018). Luego, identifica y caracteriza las principales tendencias globales. Por último, identifica las principales tendencias regionales: recesión democrática, crisis de los regionalismos y crisis de los espacios multilaterales regionales, entendiendo que éstas guardan una estrecha relación con la macro tendencia global y las tendencias internacionales.
\end{abstract}

Palabras-claves: Tendencias internacionales; Orden liberal internacional; Tablero sudamericano; Sudamérica; Orden internacional; Siglo XXI.

\begin{abstract}
The starting point of this article is that the so-called Liberal World Order is questioned by a set of global trends, among which the following stand out: the rise of China; the emergence and extension of illiberal political leadership; a certain degree of decline in free trade, in favour of less multilateral and more protectionist policies. The configuration of these global trends affects the margins of manoeuvre that South American countries have to design and execute their strategies for international insertion. For this reason, we propose to identify the most relevant global and South American trends while we assume that they constrain, and enable, affect and shape, to certain degrees and intensities, the dynamics of our region. By virtue of the foregoing, this article begins with the presentation of the foundations that account for the tensions experienced by the Liberal World Order based on the premises proposed by Haass (2018). Then identify and characterize the major global trends. Finally, it identifies the main regional trends: democratic recession, crisis of regionalisms and crisis of regional multilateral spaces, understanding that these are closely related to the global macro trend and the international trends.
\end{abstract}

Keywords: International trends; Liberal World Order; South America chessboard; 21st Century; International order. 


\section{Introducción}

Este artículo toma como punto de partida que el denominado orden liberal internacional se encuentra cuestionado. Dicho orden ha atravesado y sorteado otras situaciones de crisis en el pasado. Una de ellas en los años 1970 cuando el orden de Bretton Woods debió ser revisado y ajustado por los actores de la época. La segunda situación de crisis tuvo lugar en los años 1990 en el período inmediatamente posterior a la caída de la entonces Unión de Repúblicas Socialistas Soviéticas (URSS). La finalización del orden de Guerra Fría fue un hecho histórico que se sucedió de manera imprevista para la comunidad académica y para los hacedores de política. Y, generó un alto grado de incertidumbre, desconcierto y preocupación en torno a la idea de si era posible o no reacomodar, nuevamente, las piezas del tablero de la política internacional. Si miramos en perspectiva, es posible advertir que el orden internacional experimenta momentos de tensión cada 30 años aproximadamente.

Ahora bien, ¿cuáles son los principales aspectos de ese orden internacional que han comenzado a transitar una situación de crisis a partir de la segunda década del siglo XXI? Richard Haass (2018) lo expresa clara y sintéticamente. Las tres premisas cuestionadas del orden son: liberalismo, universalismo y la preservación del propio orden. Y, en función de ello, el autor sostiene que el orden ya no es liberal, ni internacional ni ordenado.

En consonancia con lo que afirman numerosos analistas, consideramos que las tres premisas del orden internacional liberal mencionadas están siendo puestas en tensión por un conjunto de tendencias globales entre las cuales sobresalen: el ascenso de China, la emergencia y extensión de liderazgos políticos i-liberales, cierto grado de retroceso del libre comercio en favor de políticas menos multilaterales y más proteccionistas - que afectan los flujos comerciales, de inversión y el funcionamiento de los Regionalismos -.

En ese marco, cabe preguntar por qué las tensiones del orden internacional son relevantes para Sudamérica. ${ }^{1}$ Una respuesta rápida, nos muestra que la región se encuentra inserta en ese orden internacional, que los Estados sudamericanos forman parte de la arquitectura institucional de ese orden y, en definitiva, porque en un mundo hiperconectado las tendencias globales afectan las dinámicas regionales y las características de las Políticas Exteriores de los Estados que implementan ajustes para satisfacer sus necesidades e intereses en sus vínculos con sus pares en el sistema internacional.

Una respuesta más extensa y reflexiva nos permite mostrar que el mainstream de las Teorías de las Relaciones Internacionales ha problematizado, de manera expresa o por omisión, acerca de la importancia y el rol de los factores sistémicos o externos y domésticos o internos para la política internacional y para las políticas exteriores de los Estados. En líneas generales, las Teorías Realistas y sus versiones 'neo' han priorizado los primeros mientras que la familia de Teorías de raigambre liberal, se ha concentrado sobre los segundos (MORGENTHAU, 1963; CARR, 2004; ARON, 1963; WALTZ, 1959; GILPIN, 1981; WALT, 1989; TALIAFERRO, 2009; ROSE, 1998; KEOHANE; NYE, 1977; DOYLE, 1988A; DOYLE, 1988B; MORAVCSIK, 1993; NYE, 1991; LEGRO y MORAVCSIK, 1999; IKENBERRY, 2000).

El lugar y el rol que cada teoría le asigna a los factores externos y domésticos se puede comprender atendiendo al tipo de mirada que cada una de ellas propone. Es decir, si se trata de un enfoque inside-looking out como en la mayoría de las teorías liberales o bien, outside-looking in como en la mayoría de las teorías realistas. También en el sub campo del Análisis de Política Exterior existen indicios de este debate que se inicia en la década de 1960 con la Teoría del linkage de James Rosenau (1967). Esa fue una de las obras seminales a través de la cual se comenzó a instalar la idea de que existen conexiones doméstico-externo/externo-doméstico que inciden sobre la formulación e implementación de las políticas exteriores de los Estados. En una línea similar se inscriben los trabajos de Kegley (1987); Carlsnaes (1992); Putnam (1996), Rosenau (1994); Fearon (1998); Hudson (2007); Breuning (2007); Alden y Aran (2017), entre muchos otros.

\footnotetext{
${ }^{1}$ Para un análisis detallado de la discusión Latinoamérica y Sudamérica ver por ejemplo: Samuel Pinheiro Guimarães (1999); Fernando Lorenzo e Marcel Vaillant (2005); José Antonio Sanahuja (2009), entre otros.
} 
Los académicos latinoamericanos tomaron esa idea como punto de partida y esa elección dio lugar a una extensa producción y múltiples estudios sobre las políticas exteriores de los Estados de la región (MAIRA, 1986; MUÑOZ, 1987; BUSSO, 1991; VAN KLAVEREN, 1992; RUSSELL, 1992; SOARES DE LIMA, 1994; RAPOPORT, 2004; AMORIN NETO; MALAMUD, 2015; BUSSO, 2019; CERVO, 2008).

Entonces, retomando el interrogante planteado más arriba, desde nuestra perspectiva, es relevante identificar y analizar las tendencias externas porque su configuración incide sobre los márgenes de maniobra de los que los países sudamericanos disponen para diseñar y ejecutar sus estrategias de inserción internacional. Los climas políticos, económicos, comerciales y estratégico-militares generan oportunidades y desafíos para la inserción externa de todos los actores internacionales en general y de los países sudamericanos en particular. Esto significa que los Estados tienen opciones de inserción externas disponibles que se sitúan en tiempos y espacios históricos específicos.

En el caso de este artículo, nos proponemos identificar las tendencias globales y subregionales - sudamericanas - más relevantes en tanto asumimos que las primeras constriñen, y habilitan, afectan y moldean en diversos grados e intensidades, las dinámicas de nuestra región. En esta ocasión, centramos nuestra atención sobre aquel conjunto de tendencias que consideramos han afectado con mayor intensidad a Sudamérica en las dos primeras décadas del siglo XXI. El diseño metodológico que se emplea es un abordaje bibliográfico, cualitativo, descriptivo y analítico que nos permite retomar un debate contemporáneo sobre el que el mainstream ha centrado su atención y lo articula con problemáticas regionales. Analiza el modo en el que las tendencias globales inciden sobre el escenario regional y abre un debate para nuestra comunidad académica orientado a reflexionar sobre el estado de situación de nuestra región, los márgenes de maniobra para la inserción externa, los desafíos y oportunidades que este nuevo estado de cosas representa.

El enfoque teórico adopta una perspectiva outside looking in. Se parte de los factores sistémicos, pasando por los regionales sin descuidar los aspectos políticos domésticos más relevantes. Esta decisión de focalizarnos en los factores externos, no implica desconocer la importancia y la incidencia de los factores domésticos sobre las dinámicas regionales y las políticas exteriores. De hecho, compartimos con Van Klaveren (1992), Russell (1992) y Busso (2019) que existen múltiples y diversos factores domésticos que funcionan como constreñimientos y como condición de posibilidad sobre la política exterior de los gobiernos. De la misma manera que los factores sistémicos, la importancia de los domésticos guarda relación con el tiempo y el espacio histórico.

En virtud de lo expuesto, este artículo se inicia con la presentación de los fundamentos que dan cuenta de las tensiones que experimenta el orden liberal internacional a partir de las premisas propuestas por Haass (2018). Luego, identifica y caracteriza las principales tendencias globales. Por último, identifica las principales tendencias regionales: recesión democrática, crisis de los regionalismos y crisis de los espacios multilaterales regionales, entendiendo que éstas guardan una estrecha relación con la macro tendencia global y las tendencias internacionales.

\section{La crisis del orden internacional liberal y las tendencias internacionales: una presentación estilizada}

La nueva crisis del orden internacional liberal constituye, para diversos analistas, una macro tendencia global característica de estas dos primeras décadas del siglo XXI (MEARSHEIMER, 2001; NYE, 2015; STEWART, 2017; MEARSHEIMER, 2018; LIND; WOHLFORTH, 2019; HAAS, 2018; HAASS, 2019; NYE, 2019; MEARSHEIMER, 2019; HAASS, 2020). Esta macro tendencia guarda relación con un conjunto de sucesos internacionales entre los cuales los autores coinciden en señalar el ascenso de China, la emergencia de liderazgos i-liberales que operan conjuntamente con la utilización creciente de Sharp power, un retroceso del libre mercado y un avance de tendencias proteccionistas los que serán abordados posteriormente. A partir de este cuadro de situación, presentamos de manera esquemática los principales argumentos que nos permiten comprender la actual crisis del orden internacional liberal y, luego caracterizamos las tres tendencias antes mencionadas. 
Desde la primera década del siglo XXI numerosos analistas internacionales comenzaron a expresar sus preocupaciones por la crisis que atraviesa lo que coinciden en denominar orden internacional liberal (HAASS, 2018; STEWART, 2017). En términos generales, Haass sostiene que el “[...] el debilitado orden mundial liberal no es liberal ni mundial ni ordenado" (2018, p.1) y que ello se puede entender a partir del hecho de que sus tres elementos básicos están experimentando embates a partir de las interacciones de los actores internacionales, incluyendo a los propios arquitectos del orden - la política exterior desplegada por la administración Trump en Estados Unidos es un buen ejemplo, aunque no el único -.

El primer elemento de este orden es, según Haass, el liberalismo y está siendo fuertemente cuestionado de norte a sur y de este a oeste. Los sistemas políticos democráticos, en aquellos países que han optado por dicho tipo de régimen, están atravesados por distintos tipos de crisis. En líneas generales, suele afirmarse que la democracia está amenazada por el ascenso de los populismos, independientemente del signo político e ideológico. Partidos y líderes políticos de derecha y de izquierda se las han arreglado para debilitar la tradicional división de poderes, han violado en reiteradas ocasiones el Estado de Derecho - con matices e intensidades diversos según sea el caso - y, en consecuencia, son incapaces de garantizar el respeto de las libertades, garantías y derechos de sus ciudadanos.

Cabe subrayar que, en el orden internacional, tradicionalmente, convivieron Estados con sistemas políticos democráticos y autoritarios. Pese a la relevancia de esa diferencia, los gobiernos habían logrado establecer un mínimo común denominador que solían respetar. A partir del siglo XXI, se percibe un crecimiento de las conductas de algunos gobiernos democráticos y de los i-liberales que desafían más abiertamente los cimientos del orden internacional existente, contribuyendo de ese modo a hacerlo más precario, más inestable e incierto.

El segundo elemento de este orden internacional es el universalismo. Si bien el carácter universal del orden internacional constituía más bien una aspiración de los actores internacionales que lo forjaron y que participaban de él, éstos solían esforzarse para incluir a la mayor cantidad posible de gobiernos dentro de la arquitectura institucional internacional.

Desde la segunda mitad de la década de 1990 y, particularmente después del 11-S 2001, se observa una acentuación de las prácticas unilaterales para gestionar las diversas áreas de cuestiones de la política internacional. Dicho unilateralismo ha ido en detrimento del multilateralismo tanto en las instituciones vinculadas al sistema de Naciones Unidas (ONU), a la Organización Mundial de Comercio (OMC) y a los esquemas de integración regional. Esta tendencia se refuerza a partir de la crisis financiera de 2008. A modo de ejemplo, cabe mencionar la decisión de la administración de George W. Bush de intervenir militarmente en Irak; la sucesión de intervenciones fallidas en varios Estados del Mundo Árabe en la coyuntura de la denominada Primavera; la situación de stand by en la última Ronda de negociación de la OMC; la guerra comercial entre China y Estados Unidos; la salida de Gran Bretaña de la Unión Europea -conocida como Brexit-; el resurgimiento de prácticas comerciales proteccionistas; la revisión de instrumentos comerciales bilaterales; la pérdida de vigencia de iniciativas comerciales como el Acuerdo Transpacífico (TTP) y el Acuerdo Transatlántico; los vaivenes en la iniciativa de la Ruta de la Seda; entre otras. Asimismo, el ascenso de líderes políticos i-liberales muestra un espíritu contrario al respeto y garantía de los Derechos Humanos de sus propios ciudadanos y de los individuos en el escenario internacional. En ese contexto el uso y/o abuso de sharp power es otra práctica que contribuye a erosionar los componentes liberales y universales del orden internacional.

Y, el tercer elemento del orden internacional liberal es la preservación del propio orden a través de acciones y políticas de los Estados que lo retroalimentaran de manera positiva. Esto aludía a políticas concretas basadas en el liberalismo y en el universalismo orientadas a sostener y fortalecer dicho orden como aquellas otras tendientes a adaptarlo a las características propias de los tempos históricos que se fueran sucediendo. La meta de preservación y fortalecimiento del orden liberal implicaba que se trataba de un tipo de orden con algún grado de flexibilidad para que fuese posible 'ajustarlo' sin romperlo. 
Entonces, cabe preguntar de qué manera el ascenso de China entendida como una tendencia global de estos tiempos suma tensión a ese orden internacional liberal. El ascenso de China como un actor relevante de la política internacional no es un dato nuevo. Sin embargo, la transformación de China en una potencia retadora de la primacía de Estados Unidos es un dato más reciente. Desde la década de 1990, la República Popular China (RPC) viene mostrando una gran performance económica y comercial en el tablero de la política internacional y ha desplegado diversos esfuerzos para ganar mercados en los países latinoamericanos en general y sudamericanos en particular (ELLIS, 2009; APARICIO, 2017; DUSSEL PETERS, 2015; HERRERA VINELLI, 2017; SLIPAK, 2014). En pos de ello ha propuesto diversas iniciativas económicas como inversiones en obras de infraestructura, fondos para cooperación, instalación de sedes de algunas de sus empresas en la región y, comerciales. Aunque el tamaño del mercado chino es un dato obvio, el gobierno del país despliega una política en la que se ofrece como destino para las exportaciones de distintos países, entre ellos los de nuestra región, y promueve un abanico de instrumentos comerciales para estructurar este tipo de vínculos en una situación de marcada asimetría. En el caso de los países sudamericanos las asimetrías ponen de manifiesto no sólo una cuestión de tamaño, sino también de recursos de poder a favor del gigante asiático.

La economía de la RPC experimenta desde hace varias décadas un crecimiento destacado de alrededor del 7\% en forma continua. Esto tiene un impacto sobre la demanda internacional de una canasta de productos que, también, incide sobre el precio internacional de los mismos. Durante la primera década del siglo XXI en Sudamérica tuvo lugar el denominado boom de las commodities con efectos positivos sobre las arcas de los Estados que tenían un fluido intercambio comercial con China. No obstante, los saldos de las balanzas comerciales bilaterales exhibían resultados superavitarios para la RPC, en la mayoría de los casos (SVAMPA, 2013; SVAMPA, 2016; SVAMPA, 2019). La mayor intensidad del comercio entre China y Sudamérica afectó, por ejemplo, el volumen del intercambio intra-Mercosur e intra-regional así como el desplazamiento de otros socios comerciales más tradicionales para los países de la región.

Ahora bien, el crecimiento económico de China tiene repercusiones en la política internacional. En esa dirección, es posible encontrar una nutrida literatura que plantea múltiples escenarios en el mediano plazo acerca de los caminos que la RPC podría escoger. A modo de ejemplo, existen estudios que se preguntan acerca de si el denominado ascenso pacífico del gigante asiático seguirá siendo 'pacífico' y en caso de respuestas afirmativas les preocupa por cuánto tiempo lograría mantenerse dentro de esa línea de acción. Existen otros, que parten de la sospecha y se preocupan por la posibilidad de que bajo ese supuesto pacifismo la RPC, en particular con el liderazgo político de Xi Jinping, oculte intereses más ambiciosos que aspiren a disputar el liderazgo de Estados Unidos en particular y/o de Occidente en general. En línea con ambos razonamientos, los especialistas se preguntan qué cambios serían esperables en el comportamiento internacional de la RPC y qué efectos éstos podrían tener para el resto de los actores que conforman el denominado orden internacional liberal (IKENBERRY, 2017; NYE, 2016; 2019; ACHARYA, 2017; FUKUYAMA, 2020; HAASS, 2018; MEARSHEIMMER, 2019; WALT, 2018).

En definitiva, no es el crecimiento económico de la RPC en sí mismo lo que se percibe como una amenaza creciente del orden internacional liberal sino la manera en la que se ejerce ese liderazgo político i-liberal. Las mayores preocupaciones se derivan de las posibles implicancias de una extensión del componente i-liberal en la política internacional.

Una segunda tendencia observable en el orden internacional contemporáneo es el ascenso y la coexistencia de liderazgos caracterizados como 'i-liberales'. En ese marco se inscriben líderes políticos tan disímiles e ideológicamente diversos como Donald Trump, Xi Jiping, Vladimir Putin, Kim Jong Un, Nicolás Maduro, Jair Bolsonaro entre otros. Este tipo de liderazgos preocupa a los analistas internacionales y a los hacedores de política exterior por, al menos, 3 motivos: la divergencia de cosmovisiones de los líderes liberales e i-liberales y sus impactos sobre el curso de las negociaciones internacionales multilaterales; el tipo de comportamiento que los líderes i-liberales podrían asumir en la política 
internacional y por las consecuencias que ese conglomerado de acciones más desafiantes podrían tener sobre los principios, normas y reglas del orden internacional liberal y de los regímenes internacionales asociados a éste.

Las preocupaciones relativas a la emergencia de los liderazgos i-liberales, también, se encuentran relacionadas con los postulados de la Teoría de la Paz Democrática (DOYLE, 1988a; 1988b). La raigambre kantiana de dicha teoría le permite sostener que los regímenes políticos democráticos, entendidos como democracias liberales, tenían una menor propensión a hacerse la guerra entre sí. De esa manera, la guerra se convertía en una posibilidad más remota en la medida que aumentara el número de gobiernos democráticos alrededor del globo. Asimismo, cabe subrayar que la guerra continuaba siendo una opción más probable en la que se involucrarían, en mayor medida, gobiernos no democráticos entre sí o bien, gobiernos democráticos-gobiernos no democráticos. Este postulado descansa sobre un principio articulador de la familia de teorías liberales en las Relaciones Internacionales conocido como 'analogía interna', 'inside-looking out' (BURCHILL, 2005) o innenpolitik (ROSE, 1998).

El principio de analogía interna considera que los gobiernos conducen sus vínculos externos de manera similar al modo en que lo hacen en el plano doméstico. Por un lado, los gobiernos democráticos tratan a su población como 'ciudadanos' que tienen libertades, derechos y garantías individuales que deben ser respetadas por el Estado. Incluso, los ciudadanos tienen el derecho de expresar su opinión respecto de la decisión del gobierno de su país de participar en un conflicto armado o no. Por el otro, los gobiernos no democráticos, tratan a su población como súbditos. Ese trato implica una relación de mando-obediencia mucho más jerárquica que no deja margen al disenso. Entonces, si los liderazgos iliberales se transformaran en una tendencia mayoritaria la preocupación que se configura es cuánto tardarán dichos líderes en hacerse la guerra con el riesgo adicional de que podría no tratarse sólo de una guerra convencional. A excepción de Maduro y Bolsonaro, los 4 líderes mencionados más arriba gobiernan Estados que son potencias nucleares. Esta situación resulta 'nueva' para las generaciones contemporáneas y genera un alto umbral de incertidumbre en el escenario internacional.

Un recurso que funciona conjuntamente con los liderazgos 'i-liberales' consiste en la intensificación del uso de las tecnologías 2.0 - redes sociales - no sólo con fines sociales sino también con fines instrumentales, por parte de actores públicos y privados, como una nueva modalidad de manipulación de la información y de 'guerra' - tales como guerras de desinformación, el ciberterrorismo y el ciberespionaje -. Este fenómeno es lo que Christopher Walker y Jessica Ludwig (2017) han denominado 'sharp power' o poder afilado/filoso. De acuerdo con Walker, "Sharp power has the effect of limiting free expression and distorting the political environment [...] it is called 'sharp' because it seeks 'to pierce, penetrate or perforate' the political and information environments of targeted countries” (2018, p. 12). Uno de los ejemplos más conocido del uso de sharp power fue la manipulación de los sistemas de información en las elecciones presidenciales de 2016 en Estados Unidos por parte de Rusia. Otro ejemplo es la escasa transparencia con la que diversos gobiernos democráticos e i-liberales - estarían usando los datos en el contexto de la actual pandemia por COVID 19 y las inquietudes de lo que con esos datos podrían hacer en un futuro próximo. La polémica por la conducción de la red social TikTok, supuestamente bajo control del ejército chino, es otro ejemplo en la misma dirección.

Por su parte, Joseph Nye (2018) agrega que el sharp power consiste en utilizar intencionalmente información engañosa como diseminar fake news; censurar determinada información que vaya en detrimento de la imagen del actor que emplea este recurso de poder; obtener información privada de las personas que utilizan determinadas aplicaciones en sus dispositivos móviles; espiar, spoilear y controlar el flujo de datos de determinados usuarios con propósitos hostiles y vulnerando el respeto del derecho a la privacidad. Ese uso de la información y su veloz distribución vía redes sociales se realizaría con fines perjudiciales y dañinos para las democracias aprovechando la apertura de esas sociedades libres. De acuerdo con el razonamiento de Walker y de Nye, el uso continuo del sharp power podría generar una suerte de nuevo tipo de guerra por la información llevada adelante, fundamentalmente, por Rusia y China. En otros términos, serían los líderes ‘i-liberales’ los que estarían utilizando la información engañosa como una forma de penetración en las sociedades libres 
con el objetivo de manipular la opinión pública en contra de sus gobiernos democráticos e influirla para mejorar la imagen de quiénes impulsan este tipo de acciones. Desde la perspectiva de tales autores la carencia o la insuficiencia de soft power de líderes autoritarios e i-liberales constituye el factor principal para comprender la utilización del poder afilado/filoso como medio para penetrar en otras sociedades y alcanzar sus objetivos en el escenario internacional.

La última tendencia global que se identifica, a los fines de este trabajo, consiste en la acentuación de prácticas comerciales proteccionistas razón por la cual, destacados analistas se preguntan acerca de las consecuencias que esto puede tener para el sistema multilateral de comercio, los Tratados de Libre Comercio (TLC) y los Acuerdos de Complementación Económica (ACE). Este tipo de comportamiento es fácilmente observable en las intempestivas decisiones tomadas por la administración Trump en su relación comercial con China y en la reapertura de las negociaciones del NAFTA con México. En ese marco, China ha ido adoptando respuestas reactivas de la misma naturaleza puntualmente, la imposición o suba de aranceles -. En la coyuntura actual, este tipo de prácticas comerciales -contrarias a los principios de la OMC- hace crecer la sombra del futuro por la ruptura de las reglas vigentes, así como también engendra el fantasma de una guerra comercial como la que tuvo lugar en la década de 1930 del siglo XX. Sin lugar a duda, esta mayor volatilidad de las normas en materia comercial - y en las finanzas internacionales - junto con una mayor desconfianza y pérdida de eficacia de las instituciones multilaterales genera un umbral elevado de incertidumbre en la totalidad de los Estados en general y en el escenario regional en particular.

\section{Tendencias regionales: implicancias derivadas de la crisis del orden internacional liberal}

\section{Recesión democrática: giros ideológicos y crisis políticas}

La llamada recesión democrática es una avenida de doble mano que se refuerza internamente cuando se ve validada internacionalmente. En este sentido, la primavera democrática que se vivió durante la década de 1980 y especialmente de 1990 - con la consolidación del régimen pos dictaduras militares en América Latina y la caída del comunismo -, tuvo "altibajos" hasta el presente, pero desde 1828 año en que según Huntington (1991) comienza la primera ola, es el régimen de gobierno que más se ha reproducido a nivel mundial.

Esta tendencia sudamericana, guarda relación con la tendencia internacional que hemos denominado emergencia de liderazgos políticos i-liberales. Tal como hemos señalado, en estos últimos años, el populismo en todas sus vertientes se ha convertido en una amenaza al componente liberal del orden internacional y se ha vuelto moneda corriente en la región. Y aunque el surgimiento de líderes populistas sean una amenaza a la democracia por sus tendencias al autoritarismo (WEYLAND, 2013), no se puede asimilar populismo con autoritarismo o fascismo: “(...) a populist is not yet a dictator, and a populist disfigurement of democracy remains within democratic bounds” (URBINATI, 2020). Es así como todos los líderes populistas latinoamericanos de los últimos 20 años - da Silva, Kirchner, Chávez, Morales, Bolsonaro, entre otros - llegaron al poder a través de las urnas y, salvo Maduro, todos se han ido también por el voto popular.

La particularidad en la situación actual es que como recuerda Urbinati (2020), citando al Marqués de Condorcet, “Sur la nécessité, l'excuse des tyrans”. La pandemia COVID 19 allana el camino para las medidas excepcionales, los estados de emergencia, los decretos urgentes, incluso el clamor por el cierre de los Congresos y los Poderes Judiciales. Al mismo tiempo, el populismo encuentra en sus enemigos habituales - las minorías, los inmigrantes, los opositores - una forma de validar sus discursos y justificar sus acciones en nombre del 'bien común’. Por último, se presentan tres características más que retroalimentan los malos comportamientos de los gobiernos populistas: el conflicto entre los gobiernos centrales y los gobiernos sub nacionales, en donde los primeros intentan imponer medidas a las provincias y/o municipios - esto es particularmente notable en Brasil entre Bolsonaro y los gobernadores de estados importantes como Sao Paulo o Rio de Janeiro, pero también puede observarse en México o Colombia -. La segunda remite al aplazamiento electoral siendo Bolivia el caso más saliente, ya que se posponen las elecciones presidenciales mediante un decreto del Tribunal Electoral. 
La última característica es que la pandemia ha subrayado la relevancia del Estado y su imprescindible capacidad de incidir en la realidad mediante diferentes políticas públicas (ALCÁNTARA, 2020). Todas estas características fueron y son observables en los gobiernos populistas latinoamericanos de las últimas dos décadas.

Durante la primera década del siglo XXI la mayoría de los sistemas políticos sudamericanos fueron caracterizados como populismos de izquierda. Las principales críticas estuvieron asociadas a prácticas poco transparentes en el manejo de las instituciones y de los recursos financieros del Estado, que en parte fueron usados para profundizar prácticas políticas destinadas a retener el voto popular. Asimismo, la intención de estos líderes de perpetuarse en el poder o de instalar 'herederos' también muestra un cariz populista que refuerza la idea de recesión democrática porque — para poder hacerlo- intervienen sobre los tres poderes del Estado con la finalidad de legitimar y legalizar tal perpetuación a través de reformas constitucionales.

Por otra parte, muchos de estos gobiernos fueron acusados e incluso hubo destituciones debido a las denuncias de corrupción ligadas a fraudes a través de empresas del Estado y privadas. Independientemente de que se pudieran probar o no los esquemas de corrupción en la justicia, en el siguiente turno electoral, la ciudadanía se volcó hacia líderes más conservadores, con un perfil más liberal que acentuaron en sus campañas los esquemas de corrupción como una manera eficaz de cooptar votos. Por su parte, los gobiernos liberales en general han utilizado las crisis económicas de la región asociadas a los altos niveles de corrupción como estandartes de sus campañas y la articulación de un discurso desideologizado de renovación que apela al común de la gente. En la segunda mitad de la década pasada, la región ha virado nuevamente a un neoliberalismo más ortodoxo, a las viejas políticas de ajuste fiscal, reformas laborales y privatizaciones - $\mathrm{o}$ a un recorte del gasto público si se mantiene el carácter estatal de los servicios-, pero también existen rasgos novedosos como la incorporación de CEO's del sector privado al gobierno basados en una idea de eficiencia y meritocracia.

Los líderes populistas de derecha que resultaron electos se inscribieron en un marco en donde la recesión democrática se plasmó en la securitización de la agenda de gobierno como una de las características más sobresalientes tanto al interior de los Estados como en las relaciones interestatales. Como plantea Rut Diamint (2018), los militares ya no aparecen como aliados de las fracciones perdedoras para participar de golpes de Estado contra los gobiernos constituidos, sino que lo hacen, generalmente, como parte de proyectos de seguridad pública interna. Otro fenómeno que se genera es la reactualización del rol de las Fuerzas Armadas, cada vez más identificadas con las fuerzas policiales, y la cada vez más destacada presencia de los militares en las cúpulas gubernamentales. Pero también, podemos encontrarla en otras características como son los temas fronterizos o de inmigración. La crisis estructural venezolana fue la que puso en el candelero las cuestiones migratorias. Sumado a esto, el cambio de signo político en la subregión y, en particular, el efecto mimetización del modelo de Jair Bolsonaro en términos discursivos, llevaron a algunos países de la región a asumir un discurso centrado en la aversión a la inmigración y al creciente involucramiento de las fuerzas de seguridad en temas internos. Las cuestiones migratorias como materia de seguridad nacional no tienen una raigambre identitaria en nuestros países, por lo que, en realidad, el problema es heredado. La pandemia reforzó estas tendencias y es probable que sus consecuencias se proyecten más allá de la coyuntura por la tentación de utilizar las fuerzas de seguridad en escenarios pos cuarentena y estados de emergencia.

La recesión democrática, al mismo tiempo, comprende fenómenos más coyunturales, como los giros políticos. En los últimos años, hemos asistido en América Latina al surgimiento de giros ideológicos contrapuestos entre sí. A comienzos del siglo XXI, gobiernos como el de Hugo Chávez (1999), Lula da Silva (2003), Kirchner (2003), Evo Morales (2006), Rafael Correa (2007), Tabaré Vázquez (2005), entre otros, constituyeron el primer giro a la izquierda. El segundo giro, esta vez a la derecha, comienza con el triunfo de Macri (2015), se profundiza a partir de la victoria de la oposición en las elecciones parlamentarias venezolanas (2015), la derrota de Evo Morales en el Referéndum para otro ciclo de gobierno (2016), el triunfo de Kuczynski en Perú (2016), el juicio político y destitución de Rousseff que sería reemplazada por Temer (2016), la 
asunción a las presidencias de Duque y Piñera, para finalmente consolidarse con el ascenso de Bolsonaro (2019) y de Lacalle Pou (2020). Todo esto matizado por el triunfo de Donald Trump en Estados Unidos (2016), lo que hizo que el fenómeno trascendiera los límites del debate político regional. De esta manera, el giro progresista de principios del milenio, fue contestado con un giro a la derecha a partir de 2015, y otro, aunque menos generalizado, nuevamente a la izquierda con los triunfos electorales de Alberto Fernández en Argentina y López Obrador en México.

Como han planteado anteriormente Lorenzini y Pereyra Doval (2019) la idea de 'giros' responde a que la noción introduce matices de gradualidad en términos comparados con otras categorizaciones más absolutas. Y así como Panizza (2006) afirmó que el cuadro electoral de comienzos de siglo mostró el advenimiento de una marea rosa, para ilustrar los giros políticos en la segunda década del siglo XXI, las autoras construyeron la categoría de marea celeste (LORENZINI; PEREYRA DOVAL, 2019).

Así es como, en los últimos años, dos giros políticos contrastantes se han producido en la región. Los actores políticos involucrados, las políticas implementadas y los indicadores sociales provocaron los desplazamientos del centro ideológico de los sistemas políticos regionales que se situó a la izquierda y a la derecha del centro respectivamente, y nuevamente a la izquierda en algunos países. El panorama regional muestra que la recesión democrática es un fenómeno al cual han contribuido con sus políticas tanto los gobiernos de la marea rosa como los de la marea celeste siendo el común denominador las prácticas populistas implementadas.

\section{Crisis de los regionalismos}

La crisis del componente universalista del orden liberal internacional conjuntamente con la intensificación de la guerra comercial sino-norteamericana coadyuva a un retroceso del multilateralismo, del librecambio y a un regreso de las tendencias proteccionistas. Todos esos elementos también inciden en las formas y las dinámicas que adoptan los regionalismos de América Latina. ${ }^{2}$

El siglo XXI fue testigo de varios cambios en la fisonomía de los esquemas regionales en tan sólo veinte años. Durante la década pasada, la integración y la concertación fueron agenda prioritaria en toda la región. Desde Lula y Kirchner, Brasil y Argentina respectivamente se erigieron como eje articulador de la reactivación de la integración en el MERCOSUR, bajo los parámetros del denominado Regionalismo Posliberal. Como indican las características de este último, las cuestiones políticas, ideológicas, productivas y sociales constituyeron el centro de atención de la agenda y, de alguna manera, reemplazaron el énfasis en la dimensión comercial (DA MOTTA VEIGA; RÍOS, 2007; SANAHUJA, 2012; SANAHUJA, 2014). Sanahuja plantea que este “(...) regionalismo se caracteriza por un marcado predominio de la agenda política y de seguridad, y por visiones del desarrollo críticas con el 'consenso de Washington', que han dado más importancia al papel de un Estado 'neodesarrollista', a la agenda social de la lucha contra la pobreza, la desigualdad y las asimetrías, a la adopción de políticas activas en el campo de la energía y la infraestructura, y a la cooperación 'Sur-Sur”” (2009:44). Varios mecanismos de concertación política surgieron en esta época bajo las premisas posliberales: la Unión de Naciones Suramericanas (UNASUR), la Alianza Bolivariana para los Pueblos de Nuestra América (ALBA), y la Comunidad de Estados Latinoamericanos y Caribeños (CELAC).

Asimismo, aquellos procesos de integración surgidos durante el Regionalismo Abierto de la década del noventa, como el MERCOSUR, se reformularon, a partir del Acta de Copacabana y el Consenso de Buenos Aires, con la intención de incluir objetivos sociales y productivos y dejar en el pasado al paradigma neoliberal (BRICEÑO RUIZ, 2013).

\footnotetext{
${ }^{2}$ Un factor que también podría contribuir en la comprensión de la crisis del regionalismo sería el debilitamiento de algunos vínculos bilaterales específicos. En esta ocasión, decidimos centrar nuestra atención en otros factores de carácter más general pues la inclusión del estudio de los vínculos bilaterales excede el objetivo de este trabajo.
} 
En la segunda década de esta etapa, en donde la situación internacional ya empezaba a cambiar y los estados del MERCOSUR no tenían tantos márgenes de acción, se observó una coincidencia en los discursos que priorizaron la necesidad de resolver cuestiones como las barreras al intercambio comercial que venían obstaculizando el desenvolvimiento regular. En este sentido, y a pesar de las propensiones del mercado internacional a la armonización de políticas - Regionalismo del siglo XXI -, los vaivenes y crisis políticas primaron sobre los esquemas de integración lo que hizo que los gobiernos miembros adoptaran acciones más similares a las del Regionalismo Abierto.

Paralelamente, otros Estados regionales que no forman parte del MERCOSUR mantuvieron una actitud abierta al diálogo para explorar oportunidades de negocios en esquemas más flexibles como la Alianza del Pacífico (AP) y el Acuerdo Transpacífico (TTP).

Teniendo en cuenta la renovada importancia asignada a la cuestión comercial y su regulación se observó un marcado interés por intentar ensamblar al MERCOSUR con la AP. En ese contexto se expresaron intereses por los nuevos megabloques - Tratado Transatlántico, TTP, Asociación Económica Integral Regional - que fueron visualizados como una oportunidad para hallar respuestas a las necesidades comerciales de los gobiernos de turno: mayor y mejor inserción comercial internacional, diversificación de mercados de destino y de la canasta exportadora, búsqueda de inversiones externas, entre los más destacados.

En línea con esas necesidades y la resurrección del Regionalismo Abierto, se inscribe el acuerdo entre el MERCOSUR y la Unión Europea (28 de junio 2019). Aún se desconocen en profundidad los términos de éste, pero caben algunas reflexiones, entre las cuales, quizás la más importante es la que plantea Zelicovich (2019). La autora sostiene que, en el actual contexto internacional, donde prevalecen las guerras comerciales entre los dos polos de poder más importantes, los acuerdos comerciales se constituyen en un instrumento que otorga previsibilidad ante el proteccionismo esgrimido por Trump y Xi. Asimismo, hay opiniones menos optimistas, como la del ex canciller brasileño, Celso Amorim (2019), quien afirma que la Unión Europea se ha aprovechado de la escasa capacidad negociadora del bloque sudamericano ya que Argentina y Brasil estaban fragilizados política y económicamente, y cuando se está en una situación frágil, se negocia cualquier cosa. Dicho acuerdo debe ser ratificado por el Parlamento Europeo y los legislativos de los cuatro Estados Parte del MERCOSUR. Quizás cuando ese momento llegue, el contexto global y regional podría verse modificado dificultando la materizalización de la iniciativa.

Crisis de los espacios multilaterales regionales

El universalismo multilateral surgió pos Primera Guerra Mundial como un intento de institucionalizar las relaciones interestatales y de alcanzar la paz. A medida que el orden internacional liberal se fue afianzando con el correr de los años - y especialmente con el fin de la Guerra Fría -, las instituciones multilaterales también vieron reforzadas sus capacidades y sus áreas de actuación. Sin embargo, en los umbrales del siglo XXI dichas instituciones fueron entrando en crisis y demostrando los límites del multilateralismo para solucionar problemas sin el consenso de los Estados más poderosos. Al mismo tiempo, comenzaron a surgir líderes i-liberales que cuestionaron la existencia misma de la arquitectura institucional, así como su eficacia.

En la región, el caso brasileño con Bolsonaro es el más burdo, pero no el primero ni el único que niega la importancia de la gobernanza global. Según sus ideólogos, el anti-universalismo o anti-cosmopolitismo es una manera de enfrentar la decadencia por la que está atravesando el mundo occidental y, por tanto, el desprecio del multilateralismo será interpretado por este gobierno como una medida necesaria y defensiva. Como expresa Marchao, el globalismo adapta la agenda ideológica norteamericana y de parte de Europa Occidental al escenario regional: "No Brasil, esses grupos afirmam que não são contra a globalização, mas apenas contra o que eles acreditam ser um processo de globalização influenciado e dominado por essas elites cosmopolitas e liberais internacionais” (2019). 
Desde el inicio del siglo XXI a esta parte, las instancias regionales de concertación política multilateral atravesaron diferentes etapas que reflejaron las posturas políticas de los gobernantes de turno. Así, al principio de los 2000, no sólo los Estados de la región participaron más activamente en los foros de cooperación internacional, sino que se crearon nuevas instancias. Particularmente Brasil y México fueron los países que más activos estuvieron, ya que no sólo participaron de algunos de estos grupos, sino que impulsaron su creación. Además, sus embajadores ocuparon puestos de jerarquía en la estructura institucional de los mismos.

UNASUR es el ejemplo más acabado de este comportamiento de montaña rusa. Su antecedente más inmediato tuvo lugar en el 2000 con la celebración de la Cumbre de Brasilia. Luego, los líderes de la región avanzaron en su planificación y puesta en marcha. La constitución de UNASUR tuvo lugar en 2008 y diez años después fue abandonada. El hecho de que Brasil haya sido su principal promotor y que el país haya caído en una crisis sistémica, de la cual aún no puede salir, contribuyó poco al sostenimiento de la existencia de este mecanismo multilateral regional.

En consonancia con la tendencia internacional que revela la crisis del multilateralismo, parece haberse extendido entre buena parte de los líderes populistas de derecha la creencia de que los organismos multilaterales son poco eficaces en la resolución de problemas internacionales y regionales. Esta creencia se apoya en la ineficacia que los espacios multilaterales regionales mostraron al momento de resolver situaciones de crisis. A propósito de ello, el caso venezolano, representa para los anti-universalistas, un catalizador del fracaso de UNASUR que motivó la suspensión primero y la salida después de Argentina, Brasil, Chile, Colombia, Paraguay y Perú. Esta respuesta, un tanto intempestiva, pareció desconocer los méritos de UNASUR en el manejo de las crisis de Bolivia (2008), Ecuador (2010), Paraguay (2012), e incluso Venezuela (2014-2015). El fin de UNASUR dio paso a la conformación de mecanismos de concertación más informales como el Grupo de Lima en agosto de 2017, integrado por Argentina, Brasil, Canadá, Chile, Colombia, Costa Rica, Guatemala, Honduras, México, Panamá, Paraguay y Perú. Posteriormente, se sumaron Guyana y Santa Lucía. El objetivo principal del Grupo de Lima fue buscar una salida pacífica a la crisis venezolana.

En 2019, se creó el Foro para el Progreso de América del Sur (PROSUR), promovido por Piñera y Duque. La intención subyacente en la creación de PROSUR fue reemplazar a la UNASUR. En marzo de ese año, los Presidentes de Argentina, Brasil, Chile, Colombia, Ecuador, Paraguay y Perú suscribieron la Declaración de Santiago para la Renovación y el Fortalecimiento de América del Sur que inaugura las actividades de este nuevo mecanismo multilateral regional.

Por su parte, la CELAC aún sobrevive. Heredera del Grupo Río e integrada por los países de la UNASUR más México y el Caribe. Su objetivo es generar un foro de discusión política basado en la unidad cultural sin Estados Unidos y Canadá. Creada en el apogeo del Regionalismo Posliberal; con el giro celeste sus actividades se han visto menguadas. Como plantea Duarte Gamboa (2019),

(...) la membresía de la CELAC está dividida en dos facciones. Por un lado, se encuentran los integrantes del Grupo de Lima (...) que no reconoce al régimen de Nicolás Maduro, además de apoyar la aplicación de la Carta Democrática a Nicaragua en la OEA. Por el otro, están los miembros de la ALBA, que respaldan al gobierno de Venezuela y al de Daniel Ortega en Nicaragua (...) Este enfrentamiento ideológico ha dado como resultado la cancelación de diversas reuniones sectoriales y ministeriales (2019).

En este sentido, acordamos con Herrera cuando plantea que “(...) en América Latina no se crean organizaciones regionales, sino franquicias ideológicas de los gobiernos de turno. Esa imposibilidad de crear bloques verdaderamente de integración condena de manera irremediable a la región a desempeñar un papel periférico en la escena global” (2019). Sin embargo, la proliferación de foros regionales también nos demuestra que la crisis regional no generó un colapso del multilateralismo regional, sino que metamorfoseó la concertación.

En este contexto, lejos parecen haber quedado iniciativas como el Grupo de Amigos de Venezuela (2003), la oposición conjunta a Estados Unidos para la creación del ALCA ante la propuesta brasileña de la negociación en building blocks (2005), la creación de grupos de presión como el G-20 (2003) y el NAMA-11 (2004) e incluso la participación en 
Naciones Unidas en Misiones de Estabilización como la de Haití (2004). En todas estas instancias, los países de la región tuvieron un protagonismo colectivo que fue decayendo con el correr de los años, los giros políticos y la intensificación del componente anti-universalista de ciertos líderes populistas.

\section{Reflexiones finales}

En este artículo se partió de la premisa que el orden internacional liberal se encuentra tensionado por la crisis de tres de sus elementos centrales los que, a su vez, se ven cuestionados por un conjunto de tendencias internacionales. Si bien no es posible afirmar que el orden internacional liberal ha llegado a su fin, tampoco es posible predecir la forma y las características que éste puede adquirir en el mediano plazo. El principal interrogante que surge es si las características de las tendencias internacionales contemporáneas se desintegrarán con el tiempo o si, por el contrario, su incidencia será lo suficientemente profunda como para transformarlo.

Lo que sí podemos afirmar es que, por un lado, las tendencias internacionales tienen implicancias sobre la configuración de las tendencias regionales. Por el otro, las tendencias regionales tienen un impacto más marcado sobre las dinámicas de la región cuando se hallan en consonancia con las globales que cuando las desafían. La articulación de lo global con lo regional va moldeando un zeitgeist que delimita los márgenes de acción individual y colectiva de los actores internacionales. También establece el rango de elasticidad en el que los Estados tienen "permitido o prohibido" moverse en el tablero internacional. Es decir, que generan oportunidades y desafíos para pensar las opciones de inserción internacional y regional.

A partir de este razonamiento, es posible afirmar que la crisis del componente liberal del orden internacional junto con la emergencia de los liderazgos i-liberales y el uso de sharp power configuran el cuadro de situación en el que se inscribe la tendencia regional de recesión democrática. La creencia acerca de que los populismos, de derecha o de izquierda, es un problema relativo al grado de desarrollo de los países está siendo revisada.

La crisis del universalismo implica un cuestionamiento generalizado del multilateralismo que se reproduce en el plano regional. Las crisis internacionales afectan con distintos grados de intensidad los esquemas regionales. En el caso sudamericano han sido los giros políticos los que mayor incidencia han tenido en los cambios de la agenda de los procesos de integración y en la aparición y surgimiento de esquemas de concertación política.

Los esquemas de integración económica tienen, en general, grados más altos de institucionalización lo que les permite flexibilizar sus reglas internas, pero no desaparecen porque los costos de abandonarlos resultan más elevados que los de permanecer dentro de los mismos. En cambio, los mecanismos de concertación política son más permeables a los climas políticos y, en consecuencia, quedan sujetos a la voluntad y a los intereses más coyunturales de los gobiernos de turno. A modo de ejemplo, se destaca la intención de los gobiernos de la marea rosa de institucionalizar este tipo de mecanismos como una herramienta para resguardarlos de los avatares derivados de los cambios de humor político.

Es posible observar la existencia de eventos catalizadores que abren y cierran ciclos, así como también dejan una impronta acerca de los ejes temáticos por los cuales transitará la política internacional y regional en un horizonte temporal de corto plazo. En esa dirección se subrayan los atentados del 11-S 2001, la crisis financiera de 2008, el inicio del proceso Brexit y el triunfo de Trump en 2016 y la aparición de la pandemia COVID 19 en 2020. El mundo en general y Sudámerica en particular se encuentran frente a una situación de gran incertidumbre en la que será preciso estar atentos al devenir de los acontecimientos para planificar sus estrategias de inserción internacional y regional.

\section{Referencias}

ACHARYA, Amitav. After Liberal Hegemony: The Advent of a Multiplex World Order. Ethics and International Affairs, v.31, n.3, p. 271-285, 2017. 
ALCÁNTARA, Manuel. De democracias fatigadas a democracias en cuarentena. 20 Mayo, 2020. Disponible en: https://latinoamerica21.com/de-democracias-fatigadas-a-democracias-en-cuarentena/. Acceso el 07 de agosto de 2020.

ALDEN, Chris; ARAN, Amnon. Foreign Policy Analysis. New Approaches. London and New York: Routledge and Francis and Taylor, 2017.

AMORIM, Celso. La oposición en Brasil recibe con recelos el acuerdo UE-Mercosur. Agencia EFE. Julio 1, 2019. Disponible en: https://www.efe.com/efe/america/economia/la-oposicion-en-brasil-recibe-con-recelos-el-acuerdo-ue-mercosur/20000011-4013621. Acceso el 15 de agosto de 2020.

AMORIN NETO, Octavio; MALAMUD, Andrés. What Determines Foreign Policy in Latin America? Systemic versus Domestic Factors in Argentina, Brazil, and Mexico (1964-2008). Latin American Politics and Society, v.57, n.4, p. 1-27, 2015.

APARICIO, Mariana. El Caribe frente al mundo multipolar: Apuntes sobre el comercio y la política con Estados Unidos y la República Popular de China. CariCen. Centro de Estudios Latinoamericanos, Facultad de Ciencias Políticas y Sociales, Universidad Nacional Autónoma de México, p. 1-15, 2017.

ARON, Raymond. Paz y Guerra entre las Naciones. Revista de Occidente, Madrid, 1963.

BREUNING, Marijke. Foreign Policy Analysis: A Comparative Introduction. New York: Palgrave Macmillan, 2007.

BRICEÑO RUIZ, José. Ejes y modelos en la etapa actual de la integración económica regional en América Latina. Estudios Internacionales, v.45, n.175, p.9-39, 2013.

BURCHILL, Scott. Liberalism. In: BURCHILL, S. et al. (Eds.). Theories of International Relations. New York: Palgrave-Macmillan, 2005, p.55-83.

BUSSO, Anabella. Estados Unidos y la redemocratización latinoamericana. Los condicionantes externos. Revista Estudios Internacionales, n.94, p.260-289, 1991.

BUSSO, Anabella. El vínculo entre los condicionantes internos y la política exterio. Reflexiones sobre el caso argentino. Ciclos, v.26, n.52, p.3-32, 2019.

CARLSNAES, Walter. The Agency-Structure Problem in Foreign Policy Analysis. International Studies Quarterly, v.36, n.3, p.245-270, 1992.

CARR, Edward. La crisis de los veinte años (1919-1939). Madrid: La Catarata, 2004.

DA MOTTA VEIGA, Pedro; RÍOS, Sandra. O Regionalismo pós-liberal, na América do Sul: origens, iniciativas e dilemas. CEPAL, 82, p.1$48,2007$.

DIAMINT, Rut. ¿Quién custodia a los custodios? Democracia y uso de la fuerza en América Latina. Nueva Sociedad, N²78, p.24-35, 2018.

DOYLE, Michael. Kant, Liberal Legacies, and Foreign Affairs. Part I. Philosophy and Public Affairs, v.12, n.3, p.205-235, 1998a.

DOYLE, Michael. Kant, Liberal Legacies, and Foreign Affairs. Part II. Philosophy and Public Affairs, v.12, n.4, p.323-353, 1998b.

DUARTE GAMBOA, Eric Emmanuel. La CELAC en el nuevo escenario regional. Febrero 6, 2019. Disponible en:

http://revistafal.com/la-celac-en-el-nuevo-escenario-regional/. Acceso el 20 de agosto de 2020.

DUSSEL PETERS, Enrique. Comercio y relaciones estratégicas entre América Latina y el Caribe y la República Popular China. In: Bonilla, Adrián; Milet, Paz (Eds.). China en América Latina y el Caribe: Escenarios estratégicos subregionales. San José: FLACSOCAF, 2015, p.23-50.

ELLIS, Evan. China in Latin America: The Whats and Wherefores. Estados Unidos: Lynne Rienner Publishers. 2009.

FEARON, James. Domestic politics, Foreign Policy, and Theories of International Relations. Annual Review of Political Science, v.1, n.1, p.289-313, 1998.

FUKUYAMA, Francis. The Pandemic and Political Order. Foreign Affairs, v.99, n.4, p.26-32, 2020.

GILPIN, Robert. War and Change in world politics. Cambridge: Cambridge University Press, 1981.

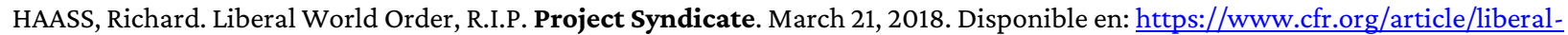
world-order-rip. Acceso el 25 de agosto de 2020. 
HAASS, Richard. How a World Order Ends? Foreign Affairs, v.98, n.1, p.22-30, 2019.

HAASS, Richard. Present at the Disruption. How Trump Unmade U. S. Foreign Policy. Foreign Affairs, v.99, n.5, p.24-34, 2020.

HERRERA VINELLI, Lorena. Latin America in Light of China's Global Economic Power: Brazil and Ecuador and Their Foreign Policies. A Comparative Study. Temas de Nuestra América. Revista de Estudios Latinoamericanos, v.33, n.61, p.89-106, 2017.

HERRERA, Juan C. Prosur, el nuevo mecanismo para no integrar Latinoamérica. The New York Times. April 9, 2019. Disponible en: https://www.nytimes.com/es/2019/04/09/espanol/opinion/prosur-unasur-america-latina.html. Acceso el 16 de agosto de 2020.

HUDSON, Valerie. Foreign Policy Analysis: Classic and Contemporary Theory. Lanham. MD: Rowman \& Littlefield, 2007.

HUNTINGTON, Samuel. La tercera ola democratizadora. Buenos Aires: Paidós, 1991.

IKENBERRY, John. After Victory. Institutions, Strategic Restraint and the Rebuilding of Order after Major Wars. Princeton: Princeton University Press, 2000.

IKENBERRY, John. The Plot Against America Foreign Policy. Can the Liberal Order Survive? 2017. Foreign Affairs. Disponible en https://www.foreignaffairs.com/articles/united-states/2017-04-17/plot-against-american-foreign-policy.

KEGLEY, Charles. The Domestic Sources of American Foreign Policy. New York: St Martins Press, 1987.

KEOHANE, Robert; NYE, Joseph. Power and Interdependence: World politics in transition. Boston: Little, Brown, 1977.

LEGRO, Jeffrey; MORAVCSIK, Andrew. Is Anybody Still realist? International Security, v.24, n.2, p.5-55, 1999.

LIND, Jennifer; WOHLFORTH, William. The future of liberal order is conservative. Foreign Affairs, v.98, n.2, p.70-80, 2019.

LORENZINI, María Elena; PEREYRA DOVAL, Gisela. Posneoliberalismo y después. El Centro Relativo del Sistema Político y el ascenso de la Marea Celeste en el espejo de la Política Exterior de Argentina y Chile. Revista de Ciencia Política, v.39, n.3, p.435-457, 2019.

LORENZO, Fernando y VAILLANT, Marcel (Cord). MERCOSUR and the Creation of the Free Trade Area of the Americas. Washington DC: Ed. Woodrow Wilson International Center for Scholars, 2003.

MAIRA, Luis. El sistema internacional y América Latina. ¿Una nueva era de hegemonía norteamericana. Buenos Aires: RIALGrupo Editor Latinoamericano, 1986.

MARCHAO, Talita. O que é o globalismo, em debate pelo Itamaraty, segundo oito especialistas. UOL Notícias. Mayo $27,2019$. Disponible en: https://noticias.uol.com.br/internacional/ultimas-noticias/2019/05/24/o-que-e-globalismo-que-sera-debatido-peloitamaraty.htm. Acceso el 10 de agosto de 2020.

MEARSHEIMER, John. The tragedy of Great Power Politics. New York: W. W. Norton Company, 2001.

MEARSHEIMER, John. The Great Delusion: Liberal Dreams and International Realities. New Haven and London: Yale University Press, 2018.

MEARSHEIMER, John. Bound to Fail: The Rise and Fall of the Liberal International Order. International Security, v.43, n.4, p.7-50, 2019.

MORAVCSIK, Andrew. Integrating International and Domestic Theories of International Bargaining. En P. Evans; H. Jacobson; R. Putnam (Eds). Double Edged Diplomacy. International Bargaining and Domestic Politics. Los Ángeles: University of California, 1993.

MORGENTHAU, Hans. La lucha por el poder y por la paz. Buenos Aires: Sudamericana, 1963.

MUÑOZ, Heraldo. Las políticas exteriores de América Latina y el Caribe: Continuidad en la crisis. Anuario de Políticas Exteriores Latinoamericanas 1986. Buenos Aires: Grupo Editor Latinoamericano-PROSPEL, 1987.

NYE, Joseph. La naturaleza cambiante del poder. Buenos Aires: Grupo Editor Latinoamericano, 1991.

NYE, Joseph. Is the American Century Over? Political Science Quarterly, v.130, n.3, p.393-400, 2015.

NYE, Joseph. Will Liberal Order Survive? The History of an Idea. Foreign Affairs. 12 de diciembre, 2016.

NYE, Joseph. China's Soft and Sharp Power. Project Syndicate. 4 de enero, 2018. Disponible en https://www.projectsyndicate.org/commentary/china-soft-and-sharp-power-by-joseph-s--nye-2018-01?barrier=accesspaylog. Acceso 25 de agosto de 2020. 
NYE, Joseph. Do morals matter? Presidents and Foreign Policy form FDR to Trump. New York: Oxford University Press, 2019.

PANIZZA, Francisco. La Marea Rosa. Correo Americano, v.8, p.5-24, 2006.

PINHEIRO GUIMARÃES, Samuel. 500 anos de Periferia. Uma contribuição ao estudo da política internacional. Porto Alegre: Ed. da Universidade, 1999.

PUTNAM, Robert. Diplomacia y política nacional: la lógica de los juegos de doble nivel. Zona Abierta, n.74, 1996.

RAPOPORT, Mario. Historia Política, Económica y Social de la Argentina (1980-2003). Buenos Aires: Ariel, 2004.

ROSE, Gideon. Neoclassical Realism and Theories of Foreign Policy. World Politics, v.51, n.1, p.144-172, 1998.

ROSENAU, James. Domestic Sources of Foreign Policy. Londres: Collier-Macmillan, 1967.

ROSENAU, James. Preteorías y teorías sobre política exterior. En Vázquez, John (Ed.) Relaciones Internacionales. El pensamiento de los clásicos. México: Ed. Limusa, 1994, p.207-2019.

RUSSELL, Roberto. Enfoques teóricos y metodológicos para el estudio de la política exterior. Buenos Aires: Grupo Editor Latinoamericano, 1992.

SANAHUJA, José Antonio. Del 'regionalismo abierto' al 'regionalismo post-liberal'. Crisis y cambio en la integración regional en América Latina. Anuario de Integración, v.7, p.12-54, 2009.

SANAHUJA, José Antonio. Enfoques diferenciados y marcos comunes en el regionalismo latinoamericano: alcance y perspectivas de UNASUR y CELAC. Pensamiento Propio, v.19, n.39, p.75-108, 2014.

SANAHUJA, José Antonio. Regionalismo posliberal y multilateralismo en Sudámerica: el caso de Unasur. Anuario de Integración, v.9, p.19-71, 2012.

SANAHUJA, José Antonio. Del "regionalismo abierto" al regionalismo post-liberal. Crisis y cambio en la integración regional en América Latina y el Caribe. CRIES, v.7, p.11-54, 2009.

SLIPAK, Ariel. América Latina y China: ¿cooperación sur-sur o consenso de Beijing? Nueva Sociedad, n.250, p.102-113, 2014.

STEWART, Patrick. Trump and the Liberal Order. The Return of Self-Help. Foreign Affairs, 13 de febrero 2017.

SVAMPA, Maristella. 'Consenso de los Commodities' y lenguajes de valoración en América Latina. Nueva Sociedad, n.244, p.30-46, 2013.

SVAMPA, Maristella. América Latina: Fin de ciclo y populismos de alta intensidad. In: GUDYNAS, Eduardo; at al. Rescatar la esperanza. Más allá del neoliberalismo y del progresismo. Barcelona: Entre Pueblos, 2016, p.63-88.

SVAMPA, Maristella. Posprogresismos, polarización y democracia en Argentina y Brasil. Nueva Sociedad, n.282, p.121-134, 2019.

TALIAFERRO, Jeffrey; LOBELL, Steven; RIPSMAN, Norris. Introduction: Neoclassical realism, the state and foreign policy. In: Lobell, Steven; Ripsman, Norris; Taliaferro, Jeffrey (Eds.). Neoclassical Realism, the State and Foreign Policy. Cambridge: Cambridge University Press, 2009, p.1-41.

URBINATI, Nadia. The Pandemic Hasn't Killed Populism. Foreign Affairs. August 6, 2020. Disponible en: https://www.foreignaffairs.com/articles/united-states/2020-08-06/pandemic-hasnt-killed-populism. Acceso el 10 de agosto de 2020.

VAN KLAVEREN, Alberto. Entendiendo las Políticas exteriores latinoamericanas: modelo para armar. Revista Estudios Internacionales, n.98, p.169-216, 1992.

WALKER, Christopher; LUDWIG, Jessica. The Meaning of Sharp Power: How Authoritarian States Project Influence. Foreign Affairs. November 16, 2017.

WALKER, Christopher. What is Sharp Power? Journal of Democracy, v.29, n.3, p.9-23, 2018.

WALT, Stephen. The Origins of Alliances. Ithaka: Cornell University Press, 1989.

WALT, Stephen. The Heel of Good Intentions. America's Foreign Policy Elite and the Decline of U. S. Primacy. New York: FarrarStraus-Giroux, 2018.

WALTZ, Kenneth. El hombre, el estado y la guerra. Buenos Aires: Editorial Nova, 1959 
WEYLAND, Kurt. The Threat from the Populist Left. Journal of Democracy, v.24, n.3, p.18-32, 2013.

ZELICOVICH, Julieta. La "Guerra Comercial” como desafío al orden liberal internacional. Anuario en Relaciones Internacionales. Disponible en: http://www.iri.edu.ar/wp-content/uploads/2019/09/a2019economiaArticulosZelicovich-1.pdf. Acceso el 15 de agosto de 2020

Funciones de colaboración ejercidas

Gisela Pereyra Doval

Maria Elena Lorenzini
Concepción; Metodología; Análisis formal; Investigación; Supervisión; Redacción(borrador original); Redacción (revisión y edición);

Concepción; Metodología; Análisis formal; Investigación; Supervisión; Redacción(borrador original); Redacción (revisión y edición); 